\title{
Veblen Hierarchy
}

\author{
Grzegorz Bancerek \\ Białystok Technical University \\ Poland
}

\begin{abstract}
Summary. The Veblen hierarchy is an extension of the construction of epsilon numbers (fixpoints of the exponential map: $\omega^{\epsilon}=\epsilon$ ). It is a collection $\varphi_{\alpha}$ of the Veblen Functions where $\varphi_{0}(\beta)=\omega^{\beta}$ and $\varphi_{1}(\beta)=\epsilon_{\beta}$. The sequence of fixpoints of $\varphi_{1}$ function form $\varphi_{2}$, etc. For a limit non empty ordinal $\lambda$ the function $\varphi_{\lambda}$ is the sequence of common fixpoints of all functions $\varphi_{\alpha}$ where $\alpha<\lambda$.

The Mizar formalization of the concept cannot be done directly as the Veblen functions are classes (not (small) sets). It is done with use of universal sets (Tarski classes). Namely, we define the Veblen functions in a given universal set and $\varphi_{\alpha}(\beta)$ as a value of Veblen function from the smallest universal set including $\alpha$ and $\beta$.
\end{abstract}

MML identifier: ORDINAL6, version: $\underline{7.11 .07 \quad 4.156 .1112}$

The papers [16], [18], [2], [5], [14], [13], [9], [10], [15], [3], [4], [1], [8], [11], [19], [17], [6], [7], and [12] provide the terminology and notation for this paper.

\section{Preliminaries}

For simplicity, we adopt the following convention: $\alpha, \beta, \gamma, \delta$ denote ordinal numbers, $\lambda$ denotes a non empty limit ordinal ordinal number, $A$ denotes a non empty ordinal number, $e$ denotes an element of $A, X, Y, x, y$ denote sets, and $n$ denotes a natural number.

Next we state several propositions:

(1) Let $\varphi$ be a function. Suppose $\varphi$ is an isomorphism between $\subseteq_{X}$ and $\subseteq_{Y}$. Let given $x, y$. If $x, y \in X$, then $x \subseteq y$ iff $\varphi(x) \subseteq \varphi(y)$.

(2) Let $X, Y$ be ordinal-membered sets and $\varphi$ be a function. Suppose $\varphi$ is an isomorphism between $\subseteq_{X}$ and $\subseteq_{Y}$. Let given $x, y$. If $x, y \in X$, then $x \in y$ iff $\varphi(x) \in \varphi(y)$. 
(3) If $\langle x, y\rangle \in \subseteq_{X}$, then $x \subseteq y$.

(4) For all transfinite sequences $f_{1}, f_{2}$ holds $f_{1} \subseteq f_{1} \frown f_{2}$.

(5) For all transfinite sequences $f_{1}, f_{2}$ holds $\operatorname{rng}\left(f_{1} \frown f_{2}\right)=\operatorname{rng} f_{1} \cup \operatorname{rng} f_{2}$.

(6) $\alpha \subseteq \beta$ iff $\varepsilon_{\alpha} \subseteq \varepsilon_{\beta}$.

(7) $\alpha \in \beta$ iff $\varepsilon_{\alpha} \in \varepsilon_{\beta}$.

Let $X$ be an ordinal-membered set. Note that $\bigcup X$ is ordinal.

Let $\varphi$ be an ordinal yielding function. Observe that $\operatorname{rng} \varphi$ is ordinal-membered.

Let us consider $\alpha$. Note that $\operatorname{id}_{\alpha}$ is transfinite sequence-like and ordinal yielding.

Let us consider $\alpha$. Observe that $\mathrm{id}_{\alpha}$ is increasing.

Let us consider $\alpha$. Note that $\operatorname{id}_{\alpha}$ is continuous.

Let us observe that there exists a sequence of ordinal numbers which is non empty, increasing, and continuous.

Let $\varphi$ be a transfinite sequence. We say that $\varphi$ is normal if and only if:

(Def. 1) $\varphi$ is an increasing continuous sequence of ordinal numbers.

Let $\varphi$ be a sequence of ordinal numbers. Let us observe that $\varphi$ is normal if and only if:

(Def. 2) $\varphi$ is increasing and continuous.

One can verify the following observations:

* every transfinite sequence which is normal is also ordinal yielding,

* every sequence of ordinal numbers which is normal is also increasing and continuous, and

* every sequence of ordinal numbers which is increasing and continuous is also normal.

Let us observe that there exists a transfinite sequence which is non empty and normal.

Next we state the proposition

(8) For every sequence $\varphi$ of ordinal numbers such that $\varphi$ is non-decreasing holds $\varphi\lceil\alpha$ is non-decreasing.

Let us consider $X$. The functor ord-type $X$ yields an ordinal number and is defined by:

(Def. 3) ord-type $X=\overline{\subseteq_{\text {On } X}}$.

Let $X$ be an ordinal-membered set. Then ord-type $X$ can be characterized by the condition:

(Def. 4) ord-type $X=\overline{\varsigma_{X}}$.

Let $X$ be an ordinal-membered set. One can verify that $\subseteq_{X}$ is well-ordering.

Let $E$ be an empty set. Observe that On $E$ is empty.

Let $E$ be an empty set. One can verify that $\bar{E}$ is empty.

Next we state four propositions: 
(9) ord-type $\emptyset=0$.

(10) ord-type $\{\alpha\}=1$.

(11) If $\alpha \neq \beta$, then ord-type $\{\alpha, \beta\}=2$.

(12) ord-type $\alpha=\alpha$.

Let us consider $X$. The functor numbering $X$ yields a sequence of ordinal numbers and is defined as follows:

(Def. 5) numbering $X=$ the canonical isomorphism between $\subseteq_{\text {ord-type } X}$ and $\subseteq_{\text {On } X}$.

Next we state four propositions:

(13) dom numbering $X=$ ord-type $X$ and $\operatorname{rng}$ numbering $X=\operatorname{On} X$.

(14) For every ordinal-membered set $X$ holds rng numbering $X=X$.

(15) Card dom numbering $X=$ Card On $X$.

(16) numbering $X$ is an isomorphism between $\subseteq_{\text {ord-type } X}$ and $\subseteq$ On $X$.

In the sequel $\varphi$ denotes a sequence of ordinal numbers.

One can prove the following propositions:

(17) If $\varphi=$ numbering $X$ and $x, y \in \operatorname{dom} \varphi$, then $x \subseteq y$ iff $\varphi(x) \subseteq \varphi(y)$.

(18) If $\varphi=$ numbering $X$ and $x, y \in \operatorname{dom} \varphi$, then $x \in y$ iff $\varphi(x) \in \varphi(y)$.

Let us consider $X$. Note that numbering $X$ is increasing.

Let $X, Y$ be ordinal-membered sets. One can check that $X \cup Y$ is ordinalmembered.

Let $X$ be an ordinal-membered set and let $Y$ be a set. Observe that $X \backslash Y$ is ordinal-membered.

The following three propositions are true:

(19) Let $X, Y$ be ordinal-membered sets. Suppose that for all $x, y$ such that $x \in X$ and $y \in Y$ holds $x \in y$. Then (numbering $X)^{\wedge}$ numbering $Y$ is an isomorphism between $\subseteq_{\text {ord-type } X \text { +ord-type } Y}$ and $\subseteq_{X \cup Y}$.

(20) For all ordinal-membered sets $X, Y$ such that for all $x, y$ such that $x \in X$ and $y \in Y$ holds $x \in y$ holds numbering $(X \cup Y)=(\text { numbering } X)^{\frown}$ numbering $Y$.

(21) For all ordinal-membered sets $X, Y$ such that for all $x, y$ such that $x \in X$ and $y \in Y$ holds $x \in y$ holds ord-type $(X \cup Y)=$ ord-type $X+$ ord-type $Y$.

\section{Fixpoints of a Normal Function}

Next we state the proposition

(27) For every function $\varphi$ such that $x$ is a fixpoint of $\varphi$ holds $x \in \operatorname{rng} \varphi$.

Let $\varphi$ be a sequence of ordinal numbers. The functor criticals $\varphi$ yields a sequence of ordinal numbers and is defined by: 
(Def. 7) $\operatorname{criticals} \varphi=$ numbering $\{\alpha \in \operatorname{dom} \varphi: \alpha$ is a fixpoint of $\varphi\}$.

We now state three propositions:

(28) $\operatorname{On}\{\alpha \in \operatorname{dom} \varphi: \alpha$ is a fixpoint of $\varphi\}=\{\alpha \in \operatorname{dom} \varphi: \alpha$ is a fixpoint of $\varphi\}$.

(29) If $x \in$ dom criticals $\varphi$, then $(\operatorname{criticals} \varphi)(x)$ is a fixpoint of $\varphi$.

(30) $\operatorname{rng}$ criticals $\varphi=\{\alpha \in \operatorname{dom} \varphi: \alpha$ is a fixpoint of $\varphi\}$ and $\operatorname{rng}$ criticals $\varphi \subseteq$ $\operatorname{rng} \varphi$.

Let us consider $\varphi$. One can verify that criticals $\varphi$ is increasing.

We now state several propositions:

(31) If $x \in \operatorname{dom}$ criticals $\varphi$, then $x \subseteq(\operatorname{criticals} \varphi)(x)$.

(32) $\operatorname{dom}$ criticals $\varphi \subseteq \operatorname{dom} \varphi$.

(33) If $\beta$ is a fixpoint of $\varphi$, then there exists $\alpha$ such that $\alpha \in \operatorname{dom}$ criticals $\varphi$ and $\beta=(\operatorname{criticals} \varphi)(\alpha)$.

(34) If $\alpha \in \operatorname{dom}$ criticals $\varphi$ and $\beta$ is a fixpoint of $\varphi$ and (criticals $\varphi)(\alpha) \in \beta$, then succ $\alpha \in$ dom criticals $\varphi$.

(35) If $\operatorname{succ} \alpha \in \operatorname{dom}$ criticals $\varphi$ and $\beta$ is a fixpoint of $\varphi$ and (criticals $\varphi)(\alpha) \in$ $\beta$, then $(\operatorname{criticals} \varphi)(\operatorname{succ} \alpha) \subseteq \beta$.

(36) Suppose $\varphi$ is normal and $\bigcup X \in \operatorname{dom} \varphi$ and $X$ is non empty and for every $x$ such that $x \in X$ there exists $y$ such that $x \subseteq y$ and $y \in X$ and $y$ is a fixpoint of $\varphi$. Then $\bigcup X=\varphi(\bigcup X)$.

(37) If $\varphi$ is normal and $\bigcup X \in \operatorname{dom} \varphi$ and $X$ is non empty and for every $x$ such that $x \in X$ holds $x$ is a fixpoint of $\varphi$, then $\bigcup X=\varphi(\bigcup X)$.

(38) If $\lambda \subseteq$ dom criticals $\varphi$ and $\alpha$ is a fixpoint of $\varphi$ and for every $x$ such that $x \in \lambda$ holds (criticals $\varphi)(x) \in \alpha$, then $\lambda \in \operatorname{dom}$ criticals $\varphi$.

(39) If $\varphi$ is normal and $\lambda \in \operatorname{dom} \operatorname{criticals} \varphi$, then $(\operatorname{criticals} \varphi)(\lambda)=$ $\bigcup(($ criticals $\varphi)\lceil\lambda)$.

Let $\varphi$ be a normal sequence of ordinal numbers. Observe that criticals $\varphi$ is continuous.

Next we state the proposition

(40) For all sequences $f_{1}, f_{2}$ of ordinal numbers such that $f_{1} \subseteq f_{2}$ holds criticals $f_{1} \subseteq$ criticals $f_{2}$.

\section{Fixpoints in a Universal Set}

In the sequel $U, W$ are universal classes.

Let us consider $U$. One can check that there exists a transfinite sequence of ordinals of $U$ which is normal.

Let us consider $U, \alpha$. An ordinal-sequence from $\alpha$ to $U$ is a function from $\alpha$ into On $U$. 
Let us consider $U, \alpha$. Note that every ordinal-sequence from $\alpha$ to $U$ is transfinite sequence-like and ordinal yielding.

Let us consider $U, \alpha$, let $\varphi$ be an ordinal-sequence from $\alpha$ to $U$, and let us consider $x$. Then $\varphi(x)$ is an ordinal of $U$.

The following two propositions are true:

(41) If $\alpha \in U$, then for every ordinal-sequence $\varphi$ from $\alpha$ to $U$ holds $\bigcup \varphi \in U$.

(42) If $\alpha \in U$, then for every ordinal-sequence $\varphi$ from $\alpha$ to $U$ holds $\sup \varphi \in U$.

In this article we present several logical schemes. The scheme CriticalNumber2 deals with a universal class $\mathcal{A}$, an ordinal $\mathcal{B}$ of $\mathcal{A}$, an ordinal-sequence $\mathcal{C}$ from $\omega$ to $\mathcal{A}$, and a unary functor $\mathcal{F}$ yielding an ordinal number, and states that:

$\mathcal{B} \subseteq \cup \mathcal{C}$ and $\mathcal{F}(\cup \mathcal{C})=\cup \mathcal{C}$ and for every $\beta$ such that $\mathcal{B} \subseteq \beta$ and $\beta \in \mathcal{A}$ and $\mathcal{F}(\beta)=\beta$ holds $\bigcup \mathcal{C} \subseteq \beta$

provided the following conditions are met:

- $\omega \in \mathcal{A}$,

- For every $\alpha$ such that $\alpha \in \mathcal{A}$ holds $\mathcal{F}(\alpha) \in \mathcal{A}$,

- For all $\alpha, \beta$ such that $\alpha \in \beta$ and $\beta \in \mathcal{A}$ holds $\mathcal{F}(\alpha) \in \mathcal{F}(\beta)$,

- Let $\alpha$ be an ordinal of $\mathcal{A}$. Suppose $\alpha$ is non empty and limit ordinal. Let $\varphi_{1}$ be a sequence of ordinal numbers. If $\operatorname{dom} \varphi_{1}=\alpha$ and for every $\beta$ such that $\beta \in \alpha$ holds $\varphi_{1}(\beta)=\mathcal{F}(\beta)$, then $\mathcal{F}(\alpha)$ is the limit of $\varphi_{1}$,

- $\mathcal{C}(0)=\mathcal{B}$, and

- For every $\alpha$ such that $\alpha \in \omega$ holds $\mathcal{C}(\operatorname{succ} \alpha)=\mathcal{F}(\mathcal{C}(\alpha))$.

The scheme CriticalNumber3 deals with a universal class $\mathcal{A}$, an ordinal $\mathcal{B}$ of $\mathcal{A}$, and a unary functor $\mathcal{F}$ yielding an ordinal number, and states that:

There exists an ordinal $\alpha$ of $\mathcal{A}$ such that $\mathcal{B} \in \alpha$ and $\mathcal{F}(\alpha)=\alpha$ provided the following requirements are met:

- $\omega \in \mathcal{A}$,

- For every $\alpha$ such that $\alpha \in \mathcal{A}$ holds $\mathcal{F}(\alpha) \in \mathcal{A}$,

- For all $\alpha, \beta$ such that $\alpha \in \beta$ and $\beta \in \mathcal{A}$ holds $\mathcal{F}(\alpha) \in \mathcal{F}(\beta)$, and

- Let $\alpha$ be an ordinal of $\mathcal{A}$. Suppose $\alpha$ is non empty and limit ordinal. Let $\varphi_{1}$ be a sequence of ordinal numbers. If $\operatorname{dom} \varphi_{1}=\alpha$ and for every $\beta$ such that $\beta \in \alpha$ holds $\varphi_{1}(\beta)=\mathcal{F}(\beta)$, then $\mathcal{F}(\alpha)$ is the limit of $\varphi_{1}$.

In the sequel $F, \varphi_{1}$ denote normal transfinite sequences of ordinals of $W$.

One can prove the following propositions:

(43) If $\omega, \beta \in W$, then there exists $\alpha$ such that $\beta \in \alpha$ and $\alpha$ is a fixpoint of $\varphi_{1}$.

(44) If $\omega \in W$, then criticals $F$ is a transfinite sequence of ordinals of $W$.

(45) If $\varphi$ is normal, then for every $\alpha$ such that $\alpha \in \operatorname{dom}$ criticals $\varphi$ holds $\varphi(\alpha) \subseteq(\operatorname{criticals} \varphi)(\alpha)$. 


\section{Sequences of Sequences of Ordinals}

Let us consider $U$ and let $\alpha, \beta$ be ordinals of $U$. Then $\alpha^{\beta}$ is an ordinal of $U$.

Let us consider $U, \alpha$. Let us assume that $\alpha \in U$. The functor $U \exp \alpha$ yields a transfinite sequence of ordinals of $U$ and is defined as follows:

(Def. 8) For every ordinal $\beta$ of $U$ holds $(U \exp \alpha)(\beta)=\alpha^{\beta}$.

Let us observe that $\omega$ is non trivial.

Let us consider $U$. Observe that there exists an ordinal of $U$ which is non trivial and finite.

One can verify that there exists an ordinal number which is non trivial and finite.

Let us consider $U$ and let $\alpha$ be a non trivial ordinal of $U$. Note that $U \exp \alpha$ is normal.

Let $\psi$ be a function. We say that $\psi$ is ordinal-sequence-valued if and only if:

(Def. 9) If $x \in \operatorname{rng} \psi$, then $x$ is a sequence of ordinal numbers.

Let $\varphi$ be a sequence of ordinal numbers. Observe that $\langle\varphi\rangle$ is ordinal-sequencevalued.

Let $\varphi$ be a function. We say that $\varphi$ has the same dom if and only if:

(Def. 10) $\operatorname{rng} \varphi$ has common domain.

Let $\varphi$ be a function. Observe that $\{\varphi\}$ has common domain.

Let $\varphi$ be a function. One can verify that $\langle\varphi\rangle$ has the same dom.

One can verify that there exists a transfinite sequence which is non empty and ordinal-sequence-valued and has the same dom.

Let $\psi$ be an ordinal-sequence-valued function and let us consider $x$. Observe that $\psi(x)$ is relation-like and function-like.

Let $\psi$ be an ordinal-sequence-valued function and let us consider $x$. Observe that $\psi(x)$ is transfinite sequence-like and ordinal yielding.

Let $\psi$ be a transfinite sequence and let us consider $\alpha$. Note that $\psi\lceil\alpha$ is transfinite sequence-like.

Let $\psi$ be an ordinal-sequence-valued function and let us consider $X$. One can check that $\psi\lceil X$ is ordinal-sequence-valued.

Let us consider $\alpha, \beta$. Observe that every function from $\alpha$ into $\beta$ is ordinal yielding and transfinite sequence-like.

Let $\psi$ be an ordinal-sequence-valued transfinite sequence. The functor criticals $\psi$ yields a sequence of ordinal numbers and is defined as follows:

(Def. 11) $\quad$ criticals $\psi=\operatorname{numbering}\left\{\alpha \in \operatorname{dom} \psi(0): \alpha \in \operatorname{dom} \psi(0) \wedge \wedge_{\varphi}(\varphi \in\right.$ $\operatorname{rng} \psi \Rightarrow \alpha$ is a fixpoint of $\varphi$ ) .

In the sequel $\psi$ is an ordinal-sequence-valued transfinite sequence.

One can prove the following propositions:

(46) Let given $\psi$. Then $\left\{\alpha \in \operatorname{dom} \psi(0): \alpha \in \operatorname{dom} \psi(0) \wedge \bigwedge_{\varphi}(\varphi \in \operatorname{rng} \psi \Rightarrow \alpha\right.$ is a fixpoint of $\varphi$ ) $\}$ is ordinal-membered. 
(47) If $\alpha \in \operatorname{dom} \psi$ and $\beta \in \operatorname{dom}$ criticals $\psi$, then (criticals $\psi)(\beta)$ is a fixpoint of $\psi(\alpha)$.

(48) If $x \in$ dom criticals $\psi$, then $x \subseteq(\operatorname{criticals} \psi)(x)$.

(49) If $\varphi \in \operatorname{rng} \psi$, then dom criticals $\psi \subseteq \operatorname{dom} \varphi$.

(50) If $\operatorname{dom} \psi \neq \emptyset$ and for every $\gamma$ such that $\gamma \in \operatorname{dom} \psi$ holds $\beta$ is a fixpoint of $\psi(\gamma)$, then there exists $\alpha$ such that $\alpha \in \operatorname{dom}$ criticals $\psi$ and $\beta=($ criticals $\psi)(\alpha)$.

(51) Suppose $\operatorname{dom} \psi \neq \emptyset$ and $\lambda \subseteq$ dom criticals $\psi$ and for every $\varphi$ such that $\varphi \in \operatorname{rng} \psi$ holds $\alpha$ is a fixpoint of $\varphi$ and for every $x$ such that $x \in \lambda$ holds (criticals $\psi)(x) \in \alpha$. Then $\lambda \in$ dom criticals $\psi$.

(52) For every $\psi$ such that $\operatorname{dom} \psi \neq \emptyset$ and for every $\alpha$ such that $\alpha \in \operatorname{dom} \psi$ holds $\psi(\alpha)$ is normal holds if $\lambda \in \operatorname{dom}$ criticals $\psi$, then $($ criticals $\psi)(\lambda)=$ U(( criticals $\psi)\lceil\lambda)$.

(53) For every $\psi$ such that $\operatorname{dom} \psi \neq \emptyset$ and for every $\alpha$ such that $\alpha \in \operatorname{dom} \psi$ holds $\psi(\alpha)$ is normal holds criticals $\psi$ is continuous.

(54) Let given $\psi$. Suppose $\operatorname{dom} \psi \neq \emptyset$ and for every $\alpha$ such that $\alpha \in \operatorname{dom} \psi$ holds $\psi(\alpha)$ is normal. Let given $\alpha, \varphi$. If $\alpha \in \operatorname{dom}$ criticals $\psi$ and $\varphi \in \operatorname{rng} \psi$, then $\varphi(\alpha) \subseteq($ criticals $\psi)(\alpha)$.

(55) Let $g_{1}, g_{2}$ be ordinal-sequence-valued transfinite sequences. If dom $g_{1}=$ $\operatorname{dom} g_{2}$ and $\operatorname{dom} g_{1} \neq \emptyset$ and for every $\alpha$ such that $\alpha \in \operatorname{dom} g_{1}$ holds $g_{1}(\alpha) \subseteq g_{2}(\alpha)$, then criticals $g_{1} \subseteq$ criticals $g_{2}$.

Let $\psi$ be an ordinal-sequence-valued transfinite sequence. The functor lims $\psi$ yielding a sequence of ordinal numbers is defined by:

(Def. 12) $\operatorname{dom} \operatorname{lims} \psi=\operatorname{dom} \psi(0)$ and for every $\alpha$ such that $\alpha \in \operatorname{dom} \operatorname{lims} \psi$ holds $(\operatorname{lims} \psi)(\alpha)=\bigcup\{\psi(\beta)(\alpha) ; \beta$ ranges over elements of $\operatorname{dom} \psi: \beta \in \operatorname{dom} \psi\}$.

Next we state the proposition

(56) Let $\psi$ be an ordinal-sequence-valued transfinite sequence. Suppose $\operatorname{dom} \psi \neq \emptyset$ and $\operatorname{dom} \psi \in U$ and for every $\alpha$ such that $\alpha \in \operatorname{dom} \psi$ holds $\psi(\alpha)$ is a transfinite sequence of ordinals of $U$. Then lims $\psi$ is a transfinite sequence of ordinals of $U$.

\section{Veblen Hierarchy}

Let us consider $x$. The functor $O S x$ yields a sequence of ordinal numbers and is defined by:

(Def. 13) $O S x=\left\{\begin{array}{l}x, \text { if } x \text { is a sequence of ordinal numbers, } \\ \text { the sequence of ordinal numbers, otherwise. }\end{array}\right.$

The functor $O S V x$ yielding an ordinal-sequence-valued transfinite sequence is defined by: 
(Def. 14) $O S V x=\left\{\begin{array}{l}x, \text { if } x \text { is an ordinal-sequence-valued transfinite sequence, } \\ \text { the ordinal-sequence-valued transfinite sequence, otherwise. }\end{array}\right.$

Let us consider $U$. The functor $U$-Veblen yields an ordinal-sequence-valued transfinite sequence and is defined by the conditions (Def. 15).

(Def. 15)(i) $\operatorname{dom}(U$-Veblen $)=$ On $U$,

(ii) $U$-Veblen $(0)=U \exp \omega$,

(iii) for every $\beta$ such that $\operatorname{succ} \beta \in \operatorname{On} U$ holds $U$-Veblen $(\operatorname{succ} \beta)=$ criticals $U$-Veblen $(\beta)$, and

(iv) for every $\lambda$ such that $\lambda \in \operatorname{On} U$ holds $U$-Veblen $(\lambda)=$ $\operatorname{criticals}(U$-Veblen $\lceil\lambda)$.

Let us note that there exists a universal class which is uncountable.

One can prove the following propositions:

(57) For every universal class $U$ holds $U$ is uncountable iff $\omega \in U$.

(58) If $\alpha \in \beta$ and $\beta, \omega \in U$ and $\gamma \in \operatorname{dom} U-\operatorname{Veblen}(\beta)$, then $U-\operatorname{Veblen}(\beta)(\gamma)$ is a fixpoint of $U$-Veblen $(\alpha)$.

(59) If $\lambda \in U$ and for every $\gamma$ such that $\gamma \in \lambda$ holds $\alpha$ is a fixpoint of $U$-Veblen $(\gamma)$, then $\alpha$ is a fixpoint of $\operatorname{lims}(U$-Veblen $\lceil\lambda)$.

(60) If $\alpha \subseteq \beta$ and $\beta, \omega \in U$ and $\gamma \in \operatorname{dom} U-\operatorname{Veblen}(\beta)$ and for every $\gamma$ such that $\gamma \in \beta$ holds $U$-Veblen $(\gamma)$ is normal, then $U$-Veblen $(\alpha)(\gamma) \subseteq$ $U$-Veblen $(\beta)(\gamma)$.

(61) Suppose $\lambda, \alpha \in U$ and $\beta \in \lambda$ and for every $\gamma$ such that $\gamma \in \lambda$ holds $U$-Veblen $(\gamma)$ is a normal transfinite sequence of ordinals of $U$. Then $(\operatorname{lims}(U-\operatorname{Veblen}\lceil\lambda))(\alpha)$ is a fixpoint of $U-\operatorname{Veblen}(\beta)$.

(62) If $\omega, \alpha \in U$, then $U$-Veblen $(\alpha)$ is a normal transfinite sequence of ordinals of $U$.

(63) If $\omega \in U$ and $U \subseteq W$ and $\alpha \in U$, then $U$-Veblen $(\alpha) \subseteq W$-Veblen $(\alpha)$.

(64) If $\omega, \alpha, \beta \in U$ and $\omega, \alpha, \beta \in W$, then $U$-Veblen $(\beta)(\alpha)=$ $W$-Veblen $(\beta)(\alpha)$.

(65) Suppose $\lambda \in U$ and for every $\alpha$ such that $\alpha \in \lambda$ holds $U$-Veblen $(\alpha)$ is a normal transfinite sequence of ordinals of $U$. Then $\operatorname{lims}(U$-Veblen $\lceil\lambda)$ is a non-decreasing continuous transfinite sequence of ordinals of $U$.

Let us consider $\alpha$. Note that $\mathbf{T}(\alpha \cup \omega)$ is uncountable.

Let us consider $\alpha, \beta$. The functor $\varphi_{\alpha}(\beta)$ yields an ordinal number and is defined by:

(Def. 16) $\varphi_{\alpha}(\beta)=\mathbf{T}(\alpha \cup \beta \cup \omega)-\operatorname{Veblen}(\alpha)(\beta)$.

Let us consider $n, \beta$. Then $\varphi_{n}(\beta)$ is an ordinal number and it can be characterized by the condition:

(Def. 17) $\varphi_{n}(\beta)=\mathbf{T}(\beta \cup \omega)$-Veblen $(n)(\beta)$.

One can prove the following propositions: 
(66) $\alpha \in \mathbf{T}(\alpha \cup \beta \cup \gamma)$.

(67) If $\omega, \alpha, \beta \in U$, then $\varphi_{\beta}(\alpha)=U$-Veblen $(\beta)(\alpha)$.

(68) $\varphi_{0}(\alpha)=\omega^{\alpha}$.

(69) $\varphi_{\beta}\left(\varphi_{\text {succ } \beta}(\alpha)\right)=\varphi_{\text {succ } \beta}(\alpha)$.

(70) If $\beta \in \gamma$, then $\varphi_{\beta}\left(\varphi_{\gamma}(\alpha)\right)=\varphi_{\gamma}(\alpha)$.

(71) $\alpha \subseteq \beta$ iff $\varphi_{\gamma}(\alpha) \subseteq \varphi_{\gamma}(\beta)$.

(72) $\alpha \in \beta$ iff $\varphi_{\gamma}(\alpha) \in \varphi_{\gamma}(\beta)$.

(73) $\varphi_{\alpha}(\beta) \in \varphi_{\gamma}(\delta)$ iff $\alpha=\gamma$ and $\beta \in \delta$ or $\alpha \in \gamma$ and $\beta \in \varphi_{\gamma}(\delta)$ or $\gamma \in \alpha$ and $\varphi_{\alpha}(\beta) \in \delta$.

\section{EPSILON NumBers}

In the sequel $U$ is an uncountable universal class.

Next we state four propositions:

(74) $U$-Veblen $(1)=\operatorname{criticals}(U \exp \omega)$.

(75) $\varphi_{1}(\alpha)$ is epsilon.

(76) For every epsilon ordinal number $e$ there exists $\alpha$ such that $e=\varphi_{1}(\alpha)$.

(77) $\varphi_{1}(\alpha)=\varepsilon_{\alpha}$.

\section{REFERENCES}

[1] Grzegorz Bancerek. Increasing and continuous ordinal sequences. Formalized Mathematics, 1(4):711-714, 1990.

[2] Grzegorz Bancerek. König's theorem. Formalized Mathematics, 1(3):589-593, 1990.

[3] Grzegorz Bancerek. The ordinal numbers. Formalized Mathematics, 1(1):91-96, 1990.

[4] Grzegorz Bancerek. Sequences of ordinal numbers. Formalized Mathematics, 1(2):281290, 1990.

[5] Grzegorz Bancerek. Tarski's classes and ranks. Formalized Mathematics, 1(3):563-567, 1990.

[6] Grzegorz Bancerek. The well ordering relations. Formalized Mathematics, 1(1):123-129, 1990.

[7] Grzegorz Bancerek. Zermelo theorem and axiom of choice. Formalized Mathematics, 1(2):265-267, 1990.

[8] Grzegorz Bancerek. Epsilon numbers and Cantor normal form. Formalized Mathematics, 17(4):249-256, 2009, doi: 10.2478/v10037-009-0032-8.

[9] Czesław Byliński. Functions and their basic properties. Formalized Mathematics, 1(1):5565, 1990.

[10] Czesław Byliński. Functions from a set to a set. Formalized Mathematics, 1(1):153-164, 1990.

[11] Czesław Byliński. Partial functions. Formalized Mathematics, 1(2):357-367, 1990.

[12] Czesław Byliński. Some basic properties of sets. Formalized Mathematics, 1(1):47-53, 1990.

[13] Agata Darmochwał. Finite sets. Formalized Mathematics, 1(1):165-167, 1990.

[14] Bogdan Nowak and Grzegorz Bancerek. Universal classes. Formalized Mathematics, 1(3):595-600, 1990.

[15] Karol Pąk. The Nagata-Smirnov theorem. Part I. Formalized Mathematics, 12(3):341346,2004

[16] Piotr Rudnicki and Andrzej Trybulec. Abian's fixed point theorem. Formalized Mathematics, 6(3):335-338, 1997. 
[17] Zinaida Trybulec. Properties of subsets. Formalized Mathematics, 1(1):67-71, 1990.

[18] Tetsuya Tsunetou, Grzegorz Bancerek, and Yatsuka Nakamura. Zero-based finite sequences. Formalized Mathematics, 9(4):825-829, 2001.

[19] Edmund Woronowicz. Relations and their basic properties. Formalized Mathematics, 1(1):73-83, 1990.

Received October 18, 2010 